\title{
Global integration of the Schrödinger equation within the wave operator formalism: The role of the effective Hamiltonian in multidimensional active spaces
}

\author{
Georges Jolicard $\dagger$, Arnaud Leclerc§, David Viennot $\dagger$, and \\ John P. Killingbeck $\ddagger$ \\ † Institut Utinam, UMR CNRS 6213, Université de Franche-Comté, \\ Observatoire de Besançon, 41 bis avenue de l'Observatoire, BP1615, 25010 \\ Besançon Cedex, France \\ $\S$ SRSMC, UMR CNRS 7565, Université de Lorraine, 1 Bd Arago 57070 Metz, \\ France \\ ‡School of Engineering, Robert Blackburn Building, University of Hull, Hull \\ HU6 7RX, UK \\ E-mail: georges.jolicard@utinam.cnrs.fr, \\ arnaud.leclerc@univ-lorraine.fr, david.viennot@utinam.cnrs.fr, \\ j.p.killingbeck@hull.ac.uk
}

\begin{abstract}
.
A global solution of the Schrödinger equation, obtained recently within the wave operator formalism for explicitly time-dependent Hamiltonians [J. Phys. A: Math. Theor. 48, 225205 (2015)], is generalized to take into account the case of multidimensional active spaces. An iterative algorithm is derived to obtain the Fourier series of the evolution operator issuing from a given multidimensional active subspace and then the effective Hamiltonian corresponding to the model space is computed and analysed as a measure of the cyclic character of the dynamics. Studies of the laser controlled dynamics of diatomic models clearly show that a multidimensional active space is required if the wavefunction escapes too far from the initial subspace. A suitable choice of the multidimensional active space, including the initial and target states, increases the cyclic character and avoids divergences occuring when one-dimensional active spaces are used. The method is also proven to be efficient in describing dissipative processes such as photodissociation.
\end{abstract}


PACS numbers: 31.15.p, 02.70.-c, 02.30.Tb, 33.80.-b 


\section{Introduction}

There are several numerical approaches for dealing with problems of quantum dynamics if a complicated time dependence is present in the Hamiltonian. Here we are particularly concerned with problems in which a molecule is subjected to an external field. In such cases, fast oscillations of the (classical) electromagnetic field and of the wavefunction must be taken into account accurately, as for example in quantum control algorithms which involve the intensive use of numerical wavefunction propagation to handle strong molecules-laser coupling [1, 2]. If the external field is a continuous wave with a constant envelope and a single well defined frequency, then the Floquet formalism is very well adapted and gives a basis of periodic solutions to the Schrödinger equation (the Floquet states) $[3,4$. In this case the problem is simplified to require only the study of a single optical period of the field. The Floquet states are generally not directly calculated in the time domain but rather via their Fourier coefficients in the frequency domain. It shall also be noted that the Floquet approach can be used to prove the equivalence of the purely quantum approach and the semi-classical one (under some precise conditions) in the strong field regime [5, 6]. The standard Floquet approach must be generalized if the field is not a continuous wave. If the intensity or the frequency of the electromagnetic field are slowly varied, good approximate results can still be obtained from the adiabatic variant of the Floquet theory [6]. However, the only exact and rigorous way to determine the wave packet evolution in the general case of a rapidly varying or chirped pulse is to use an exact wavepacket propagation. This is the topic treated here. Floquet states can still be calculated but they are called generalized Floquet states and are associated with the total duration of the interaction.

We develop a propagation method which makes use of two important ideas. The first idea is that, even in the case of a non-adiabatic or complicated pulse, timedependence can still be described by using a Fourier basis set, instead of using the differential step by step scheme adopted by some well established methods [7, 8]. Fourier grids methods have long been used to describe the molecular coordinates in wavepacket propagation [9. They have not been so widely used to treat the time dependence of wavepackets, because the real wavepacket is not generally time-periodic. We shall see that it is possible to resolve this apparent inconsistency by using absorbing 
potentials such as those introduced in ref. [10, 11]. The second important idea which we use is that the quantum states are not all equally significant in a given propagation. For example in a quantum control problem, the initial and the target states are of particular interest while in a STIRAP process three states are mostly concerned [12, etc. It is clearly appropriate to reflect this hierarchy of importance in the numerical approach used to solve the Schrödinger equation. In this article we take advantage of the subspace decomposition idea by relying on the time-dependent wave operator theory [13].

The two main ideas outlined above have been combined to propose a global integration method for the Schrödinger equation within the wave operator formalism in ref. 14. This first formulation was intended only for hermitian Hamiltonians and was limited to the use of one-dimensional active spaces; it appears to be efficient for investigating near-adiabatic evolutions. However for many processes such as dissociations, ionization and more general strong non-adiabatic interactions this first version cannot be used. It is thus necessary to generalize it: first, to be able to describe dissipative processes driven by non-hermitian Hamiltonians and second (and most importantly), to handle multidimensional active spaces for strong non-adiabatic evolutions, relevant in situations where the wavefunction escapes too far from a onedimensional model space.

In section 2 this generalization is achieved by introducing small multidimensional active spaces in place of the one-dimensional ones and by using asymptotic complex absorbing potentials to discretize correctly the interacting molecular continua. Iterative formulae are derived by combining the two techniques used in the first version of the algorithm : the Time Dependent Wave Operator (TDWO) formalism and the calculation of time-dependent integrals by using fast Fourier techniques (FFT). This leads to an iterative solution of the global dynamical problem. Low dimensional effective Hamiltonians are analysed as tools for measuring the more or less cyclic character of the dynamical processes and close relationships are revealed between this cyclic character and the relevance of using the TDWO theory. In section 3 we illustrate the algorithm and the theoretical discussion by studying a complete vibrational population transfer between the two wells of a model energy surface representing a system under the influence of laser fields. A second example describes the dissociative dynamics of the $\mathrm{H}_{2}^{+}$molecular ion coupled to a laser field. This example confirms the 
ability of the method to treat dissipative processes, if an appropriate multidimensional active space is used. Section 4 gives a discussion and some concluding remarks.

\section{Time dependent wave operator using multidimensional model spaces}

\subsection{Iterative calculation of the wave operator}

Let $\mathcal{H}$ be the Hilbert space associated with a molecular system and let $S_{o}$ be a model subspace of dimension $m$ which includes the initial molecular state. The orthogonal projector corresponding to the model space is called $P_{o}$, with $P_{o}^{2}=P_{o}, P_{o}^{\dagger}=P_{o}$, $\operatorname{tr}\left(P_{o}\right)=m$. The time-dependent wave operator associated with the two subspaces $S_{o}$ and $S(t)$ is defined as 13 :

$$
\Omega(t)=P(t)\left(P_{o} P(t) P_{o}\right)^{-1}=U(t, 0 ; H)\left(P_{o} U(t, 0 ; H) P_{o}\right)^{-1}
$$

where $P(t)$ are the projectors of the successive model spaces $S(t)$, with $S(t=$ $0)=S_{o}$ and where $U$ represents the quantum evolution operator associated with the Hamiltonian $H(t) . P(t)$ is the time-dependent transformation of $P_{o}$ under the influence of the Schrödinger equation, i.e. $P(t)=U(t, 0 ; H) P_{o} U^{\dagger}(t, 0 ; H)$. The timedependent wave operator is a generalization of the Møller wave operator and can be used to deduce the true dynamics from the dynamics within the $m$-dimensional subspace, governed by $H^{e f f}=P_{o} H \Omega$ :

$$
U(t, 0 ; H) P_{o}=\Omega(t) U\left(t, 0 ; H_{e f f}\right) .
$$

However, the wave operator exists only if the Fubini-Study distance between $P_{o}$ and $P(t)$ is small: $\operatorname{dist}_{F S}\left(P_{o}, P(t)\right)<\frac{\pi}{2}$ [15]. This means that the real dynamics should not make the wavefunction escape too far from the selected initial subspace. For strong couplings the limit value $\frac{\pi}{2}$ can easily be reached if the subspace is the one-dimensional subspace associated with the initial state [14. The choice of a multidimensional model space $S_{o}$ which includes all the states which interact strongly with the inital state can then reduce the Fubini-Study distance and make the calculation possible.

The wave operator satisfies a Bloch equation in the extended Hilbert space $\mathcal{H} \otimes L_{o}^{2}([0, T])\left(T\right.$ is the total duration of the dynamical process and $L_{o}^{2}([0, T])$ denotes the space of square integrable functions on $[0, T]$ with periodic boundary conditions),

$$
H_{F}(t) \Omega(t)=\Omega(t)(H(t) \Omega(t))=\Omega(t) H_{e f f}(t) .
$$


In the above equation $H_{F}(t)$ is the Floquet hamiltonian associated with the total duration, $H_{F}(t)=H(t)-i \hbar \partial / \partial t$. The main difficulty arising in this formalism is the integration of equation (3). Although the structure of eq. (3) looks simple, this simplicity is deceptive. First, this equation is not a pure series of instantaneous eigenequations, since the time-derivative present in $H_{F}$ in the left hand side couples together the values of $\Omega(t)$ at different times. Second, the integration of the Schrödinger equation within this formalism generally requires us to enforce the initial conditions. If the interaction is located on the finite time interval $[0, T]$ and if the initial wave function $\Psi(t=0)$ is strictly included within the subspace $S_{o}$, then the integration of equation (3) using tools such as Fourier basis sets and Fast Fourier Transform (FFT) can only give strictly T-periodic solutions. Such solutions do not generally satisfy the correct initial conditions except under special adiabatic circumstances (for example $\Psi(t=T) \propto \Psi(t=0))$. To recover the correct initial conditions by using a periodic basis set, we assume that the physical interaction is restricted to a time interval $\left[0, T_{0}\right]$, which is shorter than the total time interval $[0, T]$ used to describe it numerically, with $T_{0}<T$. Then a time-dependent absorbing potential is introduced over the artificial time extension $\left[T_{o}, T\right]$. $Q_{o}$ being the projector on the space complementary to the active space $S_{o}\left(P_{o}+Q_{o}=1\right)$, the suitable absorbing potential is [10, 11]

$$
V_{a b s}=-i V_{o p t}(t) Q_{o}
$$

where $V_{\text {opt }}(t)$ is a real positive function localised on the time interval $\left[T_{o}, T\right]$. The results of the dynamics are analysed at the final physical time $T_{o}$, the behaviour of $\Omega$ during the asymptotic time extension being purely artificial and having no influence back on the physical interval $\left[0, T_{0}\right]$.

Before going into more details about the numerical algorithm used to solve eq. (3), it is useful to clarify the general framework of the calculation. In the following the Hamiltonian $H(t)$ which drives the dynamics is the sum of an unperturbed Hamiltonian $H_{o}$, a time-dependent coupling term $V(t)$ (corresponding to the electric dipole coupling $-\vec{\mu} . \vec{E}(t)$ in laser-molecule experiments) and a timedependent absorbing potential $V_{a b s}$ (eq, 4 ). The Hilbert space $\mathcal{H}$ is assumed to be truncated to a finite-dimensional space. If the potential energy curves in $H_{o}$ are dissociative, a radial optical potential $\tilde{V}_{\text {opt }}(r)$ is introduced to discretise the continuum associated with the dissociative radial coordinate. Finally the Hamiltonian operator 
is ( $r$ is a composite molecular coordinate)

$$
H(r, t)=H_{o}(r)+V(r, t)+\tilde{V}_{o p t}(r)+V_{a b s}(t) .
$$

A complete zeroth-order basis set $\{|j\rangle\}_{j=1, N_{m}}$ is made up of $N_{m}$ eigenvectors of $H_{o}$ (or $H_{o}+\tilde{V}_{\text {opt }}(r)$ if needed). A Fourier basis set $\{|n\rangle\}_{n=1, N_{t}}$ with $\langle t \mid n\rangle=\exp (i 2 \pi n t / T$ ) is used to represent the space $L_{o}^{2}[0, T]$. Finally, if the active space is of dimension $m$, the wave operator $\Omega$ is represented by $N_{t}$ rectangular matrices whose size is $\left(N_{m} \times m\right)$ corresponding to each of the $N_{t}$ values of the Fourier frequencies (or equivalently to the $N_{t}$ discrete sampling values in time). This series of matrices can also be recast into one single $\left(\left(N_{m} \cdot N_{t}\right) \times m\right)$ rectangular matrix.

By projecting equation (3) on the left into the complementary space with projector $Q_{o}$, with $P_{o}+Q_{o}=1$, and by introducing the reduced wave operator $X=Q_{o} X P_{o}$, with $\Omega=P_{o}+X$, a new reduced equation is obtained:

$$
Q_{o}(1-X(t)) H_{F}(t)(1+X(t)) P_{o}=0
$$

where $H_{F}(t)$ includes the absorbing potential $V_{a b s}(t)$. An iterative solution of equation (6) has been proposed in [14] for a one-dimensional active space. We now derive the solution in the case of a multidimensional active space. By assuming that eq. (6) is not perfectly satisfied at the finite iteration order $(n)$, the right hand side of this equation being equal to $\Delta^{(n)}(t)=Q_{o}\left(1-X^{(n)}(t)\right) H_{F}(t)\left(1+X^{(n)}(t)\right) P_{o}$ instead of zero, one can introduce the increment $\delta X^{(n)}(t)$ such that $X^{(n+1)}(t)=X^{(n)}(t)+\delta X^{(n)}(t)$ exactly solves the equation. Expanding eq. (6) leads to:

$$
i \hbar \frac{\partial}{\partial t} \delta X^{(n)}(t)=\Delta^{(n)}(t)-\delta X^{(n)}(t) H_{\text {eff }}^{(n)}(t)+\tilde{H}_{\text {diag }}^{(n)}(t) \delta X^{(n)}(t)
$$

with

$$
\left\{\begin{array}{l}
\tilde{H}_{\text {diag }}^{(n)}(t)=Q_{o}\left[H(t)-X^{(n)}(t) H(t)\right]_{\text {diag }} Q_{o} \\
H_{\text {eff }}^{(n)}(t)=P_{o} H(t) \Omega^{(n)}(t)=P_{o} H(t)\left(P_{o}+X^{(n)}(t)\right)
\end{array}\right.
$$

To derive eq. (7), some approximations, previously tested in ref. [14] with $m=1$, have been introduced. The quadratic terms with respect to the increment $\left(\delta X^{(n)}(t)\right)$ and the non-diagonal elements of $Q_{o}\left[H(t)-X^{(n)}(t) H(t)\right] Q_{o}$ have been neglected. This leads in the multidimensional case to the following rigorous solution of equation (7),

$$
\begin{aligned}
\delta X^{(n)}(t) & =U\left(t, 0 ; \tilde{H}_{\text {diag }}^{(n)}\right) \\
& \times\left[\frac{1}{i \hbar} \int_{0}^{t} U^{-1}\left(t^{\prime}, 0 ; \tilde{H}_{\text {diag }}^{(n)}\right) \Delta^{(n)}\left(t^{\prime}\right) U\left(t^{\prime}, 0 ; H_{\text {eff }}^{(n)}\right) d t^{\prime}\right] \\
& \times U^{-1}\left(t, 0 ; H_{\text {eff }}^{(n)}\right),
\end{aligned}
$$


where the letters $U$ represent the quantum evolution operators. At a fixed time $t$, $\Delta^{(n)}(t)$ is a $\left(N_{m} \times m\right)$ rectangular matrix, $U\left(t, 0 ; \tilde{H}_{\text {diag }}^{(n)}\right)$ is a diagonal matrix with $\left(N_{m}\right)$ entries and $U\left(t, 0 ; H_{e f f}^{(n)}\right)$ is a small $(m \times m)$ matrix.

\subsection{Discrete implementation of the iterative solution}

To integrate eq. (9), a procedure based on Fast Fourier Transforms is used. A discrete finite time-grid is introduced on the time interval $[0, T]$ :

$$
t_{j}=\frac{j T}{N_{t}}, \quad j=0, \ldots, N_{t}-1
$$

together with the corresponding frequency representation:

$$
\left\{\begin{array}{l}
\nu_{j}=\frac{j}{T}, \quad j=0, \ldots, \frac{N_{t}}{2}-1 \\
\nu_{N_{t} / 2}=-\frac{N_{t}}{2 T}, \\
\nu_{j}=-\nu_{N_{t}-j}, j=\frac{N_{t}}{2}+1, \ldots, N_{t}-1
\end{array}\right.
$$

In eq.(9), the various terms are discretized by using the time-grid representation (eq. (10)). The calculation of $U\left(t_{j}, 0 ; H_{\text {eff }}^{(n)}\right)$ takes advantage of the small dimension of the $(m \times m)$ matrix $H_{\text {eff }}^{(n)}$. The evolution operator associated with $H_{\text {eff }}^{(n)}$ is calculated using

$$
U\left(t_{k}, 0 ; H_{e f f}^{(n)}\right)=\prod_{j=1}^{k} U\left(t_{j}, t_{j-1} ; H_{e f f}^{(n)}\right), k=0, \ldots, N_{t}-1 .
$$

The time evolution associated with $H_{\text {eff }}^{(n)}$ between two adjacent discrete time is approximated by

$$
U\left(t_{j}, t_{j-1} ; H_{e f f}^{(n)}\right)=\exp \left(-\frac{i}{\hbar} \int_{t_{j-1}}^{t_{j}} H_{e f f}^{(n)}\left(t^{\prime}\right) d t^{\prime}\right)
$$

and its action on an arbitrary vector is obtained by diagonalizing the matrix $\int_{t_{j-1}}^{t_{j}} H_{\text {eff }}^{(n)}\left(t^{\prime}\right) d t^{\prime}$. In eq. (9) the matrix $H_{\text {eff }}^{(n)}$ exhibits large time variations and it is essential to retain the exact expression and to make a precise calculation of $U\left(t^{\prime}, 0 ; H_{\text {eff }}^{(n)}\right)$. To do this, numerous numerical integrals are needed in eq. (13). At each iteration order $(n)$ the $m^{2}$ components $\left[H_{\text {eff }}^{(n)}\right]_{k, l}$ are integrated on the time interval $[0, T]$ by using the FFT procedure proposed in ref.[14. This method requires only two FFT to obtain the $N_{t}$ definite integrals corresponding to all the intermediate intervals $t_{j}$ in eq. (13).

The calculation of the evolution operator associated with the matrix $H_{\text {diag }}^{(n)}$ can also be done by using a Fourier algorithm. This matrix includes the asymptotic absorbing potential $V_{\text {opt }}(t)$ which can produce numerical instabilities during the 
discrete calculation of the integrals (because of its real exponential behavior). To solve this difficulty, this absorbing potential has been neglected in the term $U^{-1}\left(t^{\prime}, 0 ; \tilde{H}_{\text {diag }}^{(n)}\right)$ inside the integral $\int_{0}^{t}(\ldots)$ (c.f. eq.(9) ) and has been simultaneously preserved in the term $U\left(t, 0 ; \tilde{H}_{\text {diag }}^{(n)}\right)$ on the left in order to impose the correct initial conditions. These apparently arbitrary approximations are justified a posteriori by the convergence of the iterative solution. The matrix $\tilde{H}_{\text {diag }}^{(n)}$ is diagonal. The operator $U^{-1}\left(t^{\prime}, 0 ; \tilde{H}_{\text {diag }}^{(n)}\right)$ can be easily calculated as

$$
U^{-1}\left(t^{\prime}, 0 ; \tilde{H}_{\text {diag }}^{(n)}\right)=\exp \left(\frac{i}{\hbar} H_{o} t^{\prime}\right) \exp \left(\frac{i}{\hbar} \int_{0}^{t^{\prime}} \delta \tilde{H}_{\text {diag }}^{(n)} d t^{\prime \prime}\right) .
$$

with $\delta \tilde{H}_{\text {diag }}^{(n)}=\tilde{H}_{\text {diag }}^{(n)}-H_{o}$.

Introducing (14) in eq. (9) and using the new time-dependent matrix $\Lambda^{(n)}(t)$ defined as

$$
\Lambda^{(n)}(t)=\exp \left(\frac{i}{\hbar} \int_{0}^{t} \delta \tilde{H}_{\text {diag }}^{(n)} d t^{\prime}\right) \Delta^{(n)}(t) U\left(t, 0 ; H_{\text {eff }}^{(n)}\right),
$$

eq. (9) can be rewritten as

$$
\begin{aligned}
\delta X^{(n)}= & e^{-\frac{i}{\hbar} H_{o} t} e^{-\frac{i}{\hbar} \int_{0}^{t} \delta \tilde{H}_{\text {diag }}^{(n)} d t^{\prime}}\left(\frac{1}{i \hbar} \int_{0}^{t} e^{\frac{i}{\hbar} H_{o} t^{\prime}} \Lambda^{(n)}\left(t^{\prime}\right) d t^{\prime}\right) \\
& \times U^{-1}\left(t, 0 ; H_{\text {eff }}^{(n)}\right) .
\end{aligned}
$$

Using the Fourier transform $\tilde{\Lambda}$ of $\Lambda$, such as

$$
\Lambda^{(n)}(t)=\int_{\nu} \tilde{\Lambda}^{(n)}(\nu) \exp (i 2 \pi \nu t) d \nu
$$

eq. (16) can be rearranged in the following form

$$
\begin{aligned}
\delta X^{(n)}(t) & =\exp \left(-\frac{i}{\hbar} \int_{0}^{t} \delta \tilde{H}_{\text {diag }}^{(n)} d t^{\prime}\right) \\
& \times\left[-Z^{(n)}(t)+\exp \left(-\frac{i}{\hbar} H_{o} t\right) Z^{(n)}(t=0)\right] \\
& \times U^{-1}\left(t, 0 ; H_{\text {eff }}^{(n)}\right)
\end{aligned}
$$

where the matrix $Z^{(n)}$ is obtained by using two back and forth Fourier transforms,

$$
Z^{(n)}(t)=F T_{(t)}^{-1}\left[\frac{F T_{(\nu)}\left(\Lambda^{(n)}(t)\right)}{H_{o}+2 \pi \hbar \nu}\right] .
$$

The matrix elements of $Z^{(n)}$ in eq. (19) are calculated by using the discretized time/frequency introduced in eqs (10) and (11) and by approximating the Fourier Transforms by discrete Fast Fourier Transforms. Finally the incrementation rule

$$
X^{(n)}=X^{(n-1)}+\delta X^{(n-1)}
$$


and the equations (15), (18) and (19) constitutes the iterative scheme leading to the wave operator and to all the columns of the evolution operator (eq. (2)) issuing from subspace $S_{o}$. Any wavefunction whose initial state belongs to $S_{o}$ can be written as

$$
\left|\Psi^{(n)}(t)\right\rangle=\left(P_{o}+X^{(n)}(t)\right) U\left(t, 0 ; H_{e f f}^{(n)}\right)|\Psi(t=0)\rangle .
$$

Some approximations have been introduced within the above iterative scheme. Nevertheless the algorithm is global and these approximations cannot induce any of the cumulative errors found in a standard differential propagation scheme. The iterative procedure is stopped when the following convergence criteria is satisfied:

$$
\left\|\delta X^{(n)}\right\|^{2} /\left\|X^{(n)}\right\|^{2} \leq \epsilon
$$

where $\epsilon$ is a fixed convergence factor and $\|$.$\| denotes the Frobenius norm. The solution$ which satisfies eq. (22) is necessarily the correct and unique solution of the propagation problem with an accuracy specified by $\epsilon$.

We would like to stress the fact that a calculation with a subspace of dimension $m^{\prime}>m$ gives more results than the calculation with a subspace of dimension $m$, because more columns of the evolution operator are obtained. At the same time more columns have to be treated by the Fourier transform steps. As long as $m$ remains small this part of the calculation takes most of the CPU time. This CPU time increase is linear with $m$ and even if other parts of the calculation are not (several matrix products cost $m^{2}$ and the effective Hamiltonian diagonalizations cost $m^{3}$ ) the final CPU time increase is about linear. Moreover an increase of $m$ can produce a strong acceleration of the convergence, leading to a final decrase of the total CPU time.

\subsection{Cyclic dynamics and wave operator}

The iterative solution proposed in the previous subsection is relevant for dynamical processes in which the wavefunctions does not escape very far from the model subspace. The quantum distance between the dynamically developing subspace and the model space $S_{o}$ can be monitored by using the Fubini-Study distance between $S_{o}$ and $S(t)$, namely $\operatorname{dist}_{F S}\left(S_{o}, S(t)\right)$. This distance goes from 0 , when the two subspace are equal, and increases to reach its maximum possible value of $\pi / 2$ when the subspaces are orthogonal. This situation could make the iterative algorithm diverge. If $|i\rangle$ is the initial state and the wavefunction is $\left|\Psi_{i}(t)\right\rangle=U(t, 0 ; H)|i\rangle$, the Fubini-Study distance 
corresponding to a one-dimensional active space is simply equal to:

$$
\operatorname{dist}_{F S}\left(S_{o}, S(t)\right)=\arccos \left(\left\|\left\langle i \mid \Psi_{i}(t)\right\rangle\right\|\right)=\arccos \left(\frac{1}{\|\Omega(t)\|}\right) .
$$

Eq. (23) indicates that the limit value is reached if the survival probability vanishes at a given time, which is equivalent to say that the wave operator diverges at the same time. There are two different approaches to overcome the possible difficulty due to nearly-orthogonal subspaces. In ref. [15] time-dependent adiabatic deformations of the active space are shown to be efficient for describing almost adiabatic quantum systems. Here we adopt another point of view and we try to improve the integration scheme by using multidimensional active spaces in the global integration procedure. In the multidimensional case, the above definition of the quantum distance is generalized to give

$$
\operatorname{dist}_{F S}\left(S_{o}, S(t)\right)=\arccos \left|\operatorname{det}\left(\mathbf{P}_{\mathbf{o}}^{\dagger} \cdot\left(\mathbf{U}(\mathbf{t}, \mathbf{0} ; \mathbf{H}) \mathbf{P}_{\mathbf{o}}\right)\right)\right| .
$$

where $\mathbf{P}_{\mathbf{o}}^{\dagger}$. $\left(\mathbf{U}(\mathbf{t}, \mathbf{0} ; \mathbf{H}) \mathbf{P}_{\mathbf{o}}\right)$ designates the matrix representation of the operator $P_{o}^{\dagger} .\left(U(t, 0 ; H) P_{o}\right)$. The conditions for this distance to reach its limit value of $\pi / 2$ are less easily satisfied and the convergence of the iterative scheme becomes more robust.

In this context cyclic dynamics are a particular case of special interest. In this case the wavefunction issuing from subspace $S_{o}$ comes back to $S_{o}$ at time $T$. A cyclic dynamics, with respect to the subspace $S_{o}$ of dimension $m$, can be defined as follows: $\forall i \in[1, m]$, let $\Psi_{i}^{o}(t)$ be the solution of the Schrödinger equation with the initial condition $\Psi_{i}^{o}(t=0)=|i\rangle$, then there exists a unitary gauge transformation $W$ such that $\Psi_{i}^{o}(t)=\sum_{j=1}^{m} W_{j i}(t) \tilde{\Psi}_{j}^{o}(t)$ with $\tilde{\Psi}_{i}^{o}(0)=|i\rangle=\tilde{\Psi}_{i}^{o}(T)$. This condition implies

$$
P_{o} \Psi(T)=\Psi(T)
$$

and by taking eq. (21) into account,

$$
X^{N}(t=0)=X^{N}(t=T)=0 .
$$

This means that the TDWO is also cyclic with $\Omega(T)=\Omega(0)=P_{o}$.

For any Hamiltonian, a perfect artificial cyclic dynamics can always be obtained, independently of the $m$ value, by adding a time-dependent absorbing potential (eq.(44) on the time extension $\left[T_{o}, T\right]$. This constrained cyclicity is a numerical artefact which can be seen as the multidimensional generalization of the constrained adiabatic trajectory method (CATM) of ref. [10, 11. A true cyclic (or maybe quasi-cyclic) 
dynamics can also be obtained in particular cases by the natural evolution on $\left[0, T_{0}\right]$, without introducing any artificial absorbing potential. In the case of a natural cyclicity, eq.(26) may not be rigorously satisfied. Nevertheless, the asymptotic values $X^{N}(0)=X^{N}(T)$, even when not exactly equal to zero, may be small enough to consider the dynamics as cyclic. We will see later that such a naturally cyclic situation has beneficial consequences on the convergence of the iterative process. The main question is to select, with or without an absorbing potential, the best active space at a fixed small degeneracy, with order $m \neq 1$. In most cases the choice of the model space should be based on physical considerations, thus including the initial state, strongly coupled states and states corresponding to resonant transitions. Choosing too small a dimension $m$ may produce too large a Fubini-Study distance between $S_{o}$ and $S(t)$. Including more states in the subspace avoids such problems.

Cyclic dynamical processes belong to the framework of the non-abelian, nonadiabatic Berry phase formulation, known as the non-Abelian Aharonov-Anandan phase formulation [17]. This theory is consistent with the wave operator approach used in this article. More precisely if $\left\{\Psi_{i}^{o}(t)\right\}_{i}$ is a non-abelian parallel transport associated with the section $\left\{\tilde{\Psi}_{i}^{o}\right\}_{i}$, then $\left\{\Omega \Psi_{i}^{o}\right\}_{i}$ is a non-abelian parallel transport associated with the section $\left\{\Omega \tilde{\Psi}_{i}^{o}\right\}_{i}[16]$.

In this cyclic context, important results can be derived by considering the expansion of the wave function on the Floquet eigenstates basis set. In the extended Hilbert space $\mathcal{H} \otimes L_{o}^{2}([0, T])$, the generalized Floquet eigenstates are defined as solutions of the following equation:

$$
H_{F}\left|\lambda_{j, n}\right\rangle=E_{j, n}\left|\lambda_{j, n}\right\rangle
$$

where $H_{F}=H-i \hbar \partial / \partial t$. The label $n$ corresponds to the different Floquet blocks of eigenstates and $j$ distinguishes the states within each block (this index is associated with the molecular Hilbert space). The eigenfunctions $|\lambda\rangle$ are $T$-periodic on the full interaction interval $[0, T]$ and satisfy the orthonormality condition [18]

$$
\left\langle\left\langle\lambda_{i^{\prime}, n^{\prime}} \mid \lambda_{i, n}\right\rangle\right\rangle=\frac{1}{T} \int_{0}^{T} d t\left\langle\lambda_{i^{\prime}, n^{\prime}} \mid \lambda_{i, n}\right\rangle=\delta_{i^{\prime}, i} \delta_{n^{\prime}, n} .
$$

The wavefunction can be expanded on a complete set of Floquet eigenvectors,

$$
|\Psi(t)\rangle=\sum_{j=1}^{N_{m}} \sum_{n=0}^{N_{t}-1} e^{-i E_{j, n} t / \hbar}\left|\lambda_{j, n}(t)\right\rangle\left\langle\left\langle\lambda_{j, n} \mid \Psi(t=0)\right\rangle\right\rangle .
$$


By taking into account the periodicity of the Floquet blocks, the double summation in eq.(29) can be reduced to a unique one in the first Brillouin zone without introducing any approximation:

$$
|\Psi(t)\rangle=\sum_{j=1}^{N_{m}} e^{-i E_{j, 0} t / \hbar}\left|\lambda_{j, 0}(t)\right\rangle\left\langle\lambda_{j, 0}(t=0) \mid \Psi(t=0)\right\rangle
$$

In the case of a cyclic dynamics within a $m$-dimensional subspace, Appendix $\mathrm{A}$ demonstrates that the sum in eq.(30) is limited to only $m$ terms. The wavefunction starting at $\left|\Psi_{i}(0)\right\rangle=|i\rangle$ can be written as

$$
\left\{\begin{array}{l}
\left|\Psi_{i}(t)\right\rangle=\sum_{j=1}^{m} e^{-\frac{i}{\hbar} E_{j, 0} t}\left|\lambda_{j, 0}(t)\right\rangle U_{j i} \\
U_{j i}=\left\langle\lambda_{j, 0}(0) \mid i\right\rangle
\end{array}\right.
$$

and the $m$ relevant Floquet eigenstates have, at the two boundaries $t=0$ and $t=T$, non-vanishing components within the $S_{o}$ space exclusively,

$$
Q_{o}\left|\lambda_{j, 0}(0)\right\rangle=Q_{o}\left|\lambda_{j, 0}(T)\right\rangle=0, j=1, \ldots, m .
$$

In Appendix A we also show that the Floquet eigenvalues $E_{j, 0}$ and the $m$ components of the corresponding eigenstates within $S_{o}$ at $t=0$ can be easily derived from the time-dependent waveoperator.

In numerical examples, the comparison of the solutions of eq.(3) on the interval $\left[0, T_{o}\right]$, obtained with and without temporal absorbing potential, will give us an indication about the naturally cyclic character of the dynamics driven by $H(t)$. The absorbing potential introduced in the interval $\left[T_{o}, T\right]$ is useless if the dynamics of the wave function is purely cyclic within the finite active subspace $S_{o}$. In what follows we will select situations and laser parameters such that controlled population exchanges are obtained which are close to such naturally cyclic dynamics.

\section{Two illustrative examples}

\subsection{STIRAP in an asymmetric double-well}

Figure 1 shows two potential curves for a model diatomic molecule submitted to two laser pulses. These two curves are defined as quartic polynomials, $\epsilon_{1}(R)=$ $-5 R^{2}+0.5 R^{3}+R^{4}$ and $\epsilon_{2}(R)=0.2 R^{4}$. They can refer to two electronic states of a $1 \mathrm{D}$ vibrational Hamiltonian in the framework of the Born-Oppenheimer approximation. Similar potentials are also obtained in effective isomerization problems along a reaction coordinate 20, or to describe the nitrogen inversion within an one dimensional 
method, the asymmetry of the potential appearing when the molecule is put down on a surface. In all this subsection, arbitrary units (arb. u.) are used with $\hbar=1$ and the various numerical parameters have been adjusted to produce realistic dynamics. The dipole moment which couples $\epsilon_{1}$ with $\epsilon_{2}$ is given an arbitrary constant unit value.

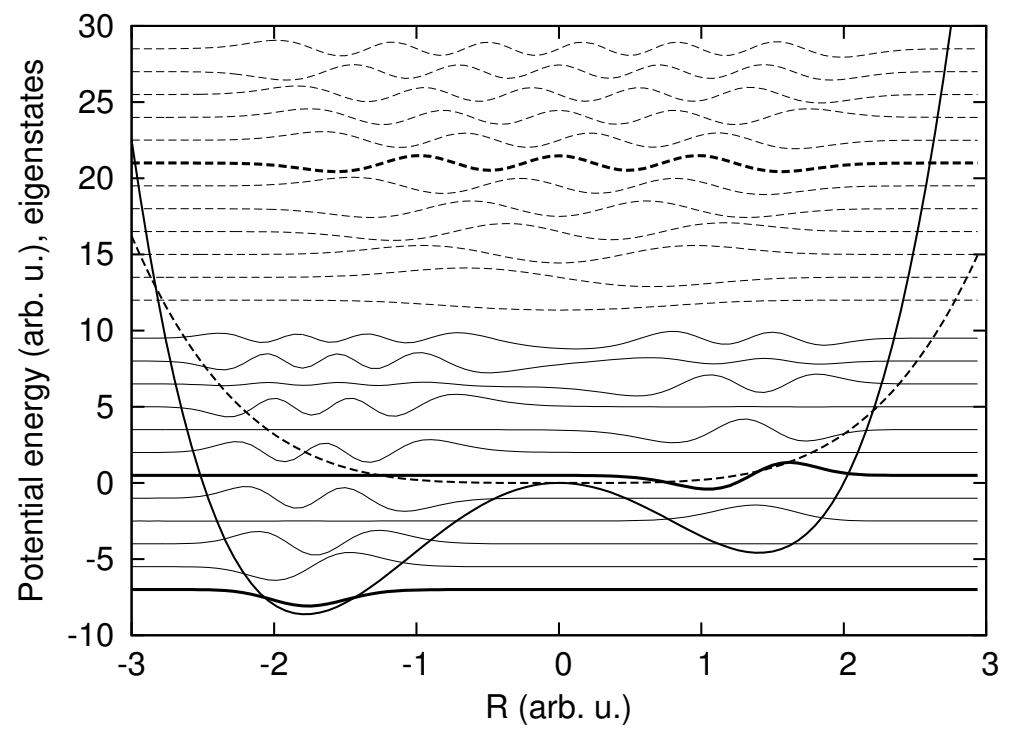

Figure 1. Potential energy curves and first vibrational eigenstates of $\epsilon_{1}(R)$ (full lines) and of $\epsilon_{2}(R)$ (dashed lines). The thick lines correspond to the initial, target and intermediate states.

The selected laser pulses are chosen to produce a complete transfer between the two asymmetric wells of the first surface. In figure 11 the vibrational state $(v=0, S=1)$ localized in the first well and the state $(v=5, S=1)$ localized in the second well have in common a strong overlap with state $(v=6, S=2)$. To obtain a stimulated Raman adiabatic passage [12, 22, the laser field which couples the two surfaces is chosen as the sum of two pulses with gaussian envelopes and carrier frequencies in resonance with the transitions $(v=0, S=1) \rightarrow(v=6, S=2)$ and $(v=6, S=2) \rightarrow(v=5, S=1):$

$$
E(t)=\sum_{j=1}^{2} E_{j} \cos \left(\omega_{j}\left(t-T_{j}\right)\right) \exp \left(-\left(\frac{t-T_{j}}{\tau_{j}}\right)^{2}\right)
$$


with the following numerical parameters

$$
\left\{\begin{array}{l}
E_{1}=0.03, \omega_{1}=4.77725153, T_{1}=250, \tau_{1}=125 \\
E_{2}=0.03, \omega_{2}=9.9844894, T_{2}=360, \tau_{2}=125
\end{array}\right.
$$

The nuclear dynamics is governed by the time-dependent Schrödinger equation within the framework of the dipole approximation.

$$
i \frac{\partial}{\partial t} U(t, 0)=\left[T_{N}+\left(\begin{array}{cc}
\epsilon_{1} & -\vec{\mu}_{1,2} \cdot \vec{E}(t) \\
-\vec{\mu}_{1,2} \cdot \vec{E}(t) & \epsilon_{2}
\end{array}\right)\right] U(t, 0)
$$

where $T_{N}$ is the relative kinetic energy of the two atoms.

This dynamics is described within a basis set made of the 30 first vibrational eigenstates for each surface (namely $N_{m}=60$ ) and $N_{t}=65536$ sampling time values (c.f. eq. 10) equally distributed over the time interval $[0, T=800]$ and with a time-dependent absorbing potential (eq. 4, subsection 2.1) localised on the time interval $[600,800]$. Thirty states per surface are sufficient to give convergence of the calculations with the selected laser amplitudes and laser frequencies. A first calculation is made with the wave operator formalism proposed in [14 by using a one-dimensional active space based on the initial state $(v=0, S=1)$. This choice produces a strong divergence of the algorithm from the start of the iterative procedure.

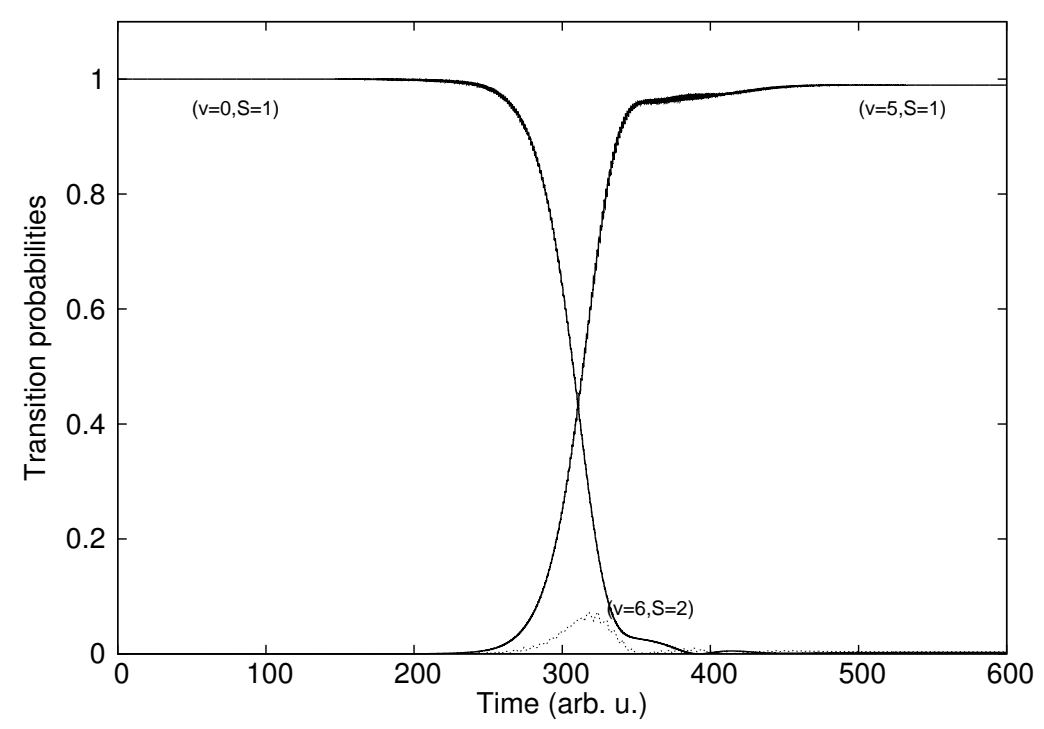

Figure 2. Evolution of the populations of the initial state, $(v=0, S=1)$, the target state $(v=5, S=1)$ and the intermediate state $(v=6, S=2)$ during the molecule-laser interaction. 


\begin{tabular}{|c|c|c|c|c|c|c|c|}
\hline \multirow{2}{*}{$\begin{array}{c}\text { Convergence } \\
\text { Factor }\end{array}$} & \multicolumn{7}{|c|}{ Iteration number } \\
\hline & $\mathrm{n}=1$ & $\mathrm{n}=2$ & $\mathrm{n}=3$ & $\mathrm{n}=4$ & $\mathrm{n}=5$ & $\mathrm{n}=6$ & $\mathrm{n}=7$ \\
\hline$F_{n}^{(m=3)}$ & $2.11 \mathrm{E}-02$ & $1.58 \mathrm{E}-03$ & $2.09 \mathrm{E}-04$ & $4.89 \mathrm{E}-04$ & $2.86 \mathrm{E}-04$ & $7.78 \mathrm{E}-04$ & $4.21 \mathrm{E}-04$ \\
\hline$F_{n}^{(m=5)}$ & $2.22 \mathrm{E}-02$ & $1.33 \mathrm{E}-03$ & 3.03 E-05 & $1.29 \mathrm{E}-06$ & $1.98 \mathrm{E}-07$ & $1.20 \mathrm{E}-07$ & $2.00 \mathrm{E}-07$ \\
\hline
\end{tabular}

Table 1. The convergence factor $F_{m}^{(n)}=\left\|\delta X^{(n)}\right\|^{2} /\left\|X^{(n)}\right\|^{2}$ (eq. (22) with respect to the iteration number $n$ for active subspaces of dimension $m=3$ and $m=5$.

We then applied the formalism of the present paper by using multidimensional active spaces of increasing dimension. A second attempt used an active space made up of two states: the initial state $(v=0, S=1)$ and the target state $(v=5, S=1)$. A third attempt involves adding the intermediate state $(v=6, S=2)$ to these two states. Finally a fourth calculation includes the quasi-resonant states $(v=16, S=2)$ and $(v=6, S=1)$ together with the three previous states to constitute an active space $S_{o}$ of dimension $m=5$.

The second choice $(m=2)$ produces, like the first one, a strong and rapid divergence of the iterative calculation. On the contrary the use of the active spaces of dimension $=3$ and $m=5$ lead to converged results, with a much better precision in the last case $m=5$. The table (11) shows the convergence factor (cf eq. (22))

In spite of a much better convergence in the case $m=5$ than in the case $m=3$ the two calculations give undistinguishable results in figure 2 with an almost complete transfer of population. We note that the transfer is not perfectly complete $\left(P(t=T)_{v=0 \rightarrow v=5}=0.9896\right)$ and that the occupation of the intermediate state $(v=6, S=2)$ is not strictly equal to zero during the interaction.

The drastically different behaviours observed during the iterative process when the dimension $m$ changes can be understood by analysing the corresponding FubiniStudy distances [21] between the active spaces at the initial instant $t=0$ and at the current time $t$ (see figure 3). The maximum value of $\pi / 2$ associated with the notion of quantum incompability of active spaces is reached in the non-degenerate case $(m=1)$ when the population of the initial state tends to zero, i.e. when the complete population transfer between $(v=0, S=1)$ and $(v=5, S=1)$ is achieved. This limit produces the divergence of the one dimensional wave operator, since $\|\Omega(t)\|=$ $\|1 /\langle i \mid \Psi(t)\rangle\|$ and indirectly that of the effective Hamiltonian $H_{e f f}(t)=P_{o} H(t) \Omega(t)$ 


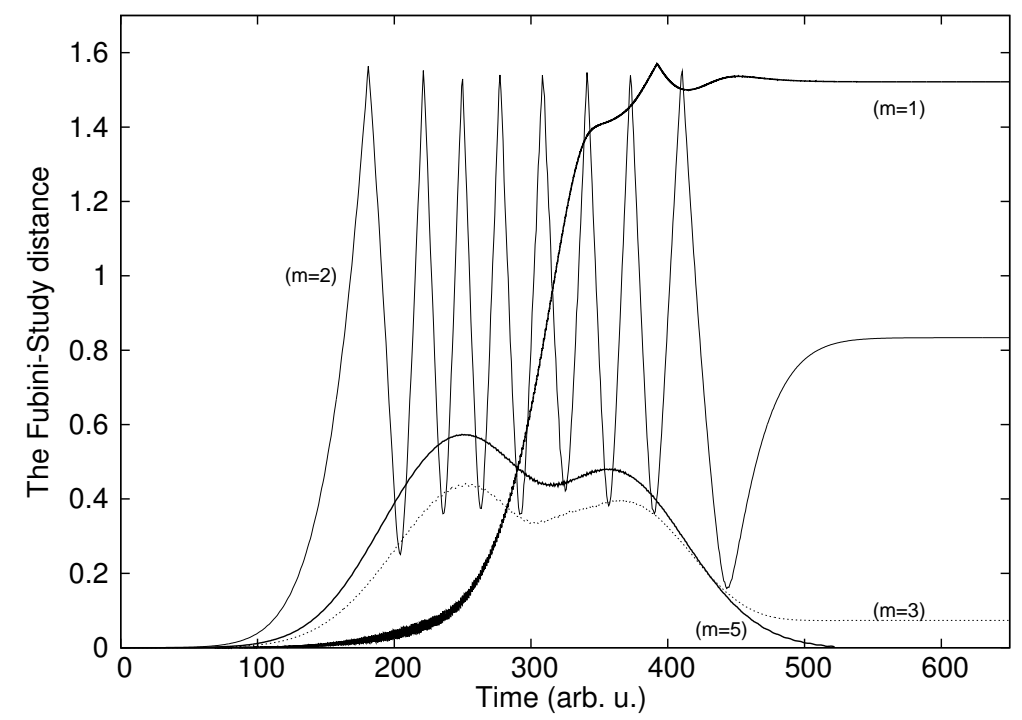

Figure 3. Fubini-Study distance (24) as a function of time by using a onedimensional active space including only the initial state $(v=0, S=1)$ (broad full line with a plateau at about $\pi / 2$ for $t \geq 400$ ), a multidimensional active space of dimension $m=2$ including the initial state and the target state $(v=5, S=1)$ (full line), a multidimensional active space of dimension $(m=3)$ obtained by adding the intermediate state $(v=6, S=2)$ to the two previous states (dashed line) and finally an active space of dimension $m=5$ by adding to the three preceding states the states $(v=16, S=2)$ and $(v=6, S=1)$ (broad full line).

which drives the dynamics within the active subspace. On the contrary one can observe in figure 3 that, in the $m=3$ and $m=5$ cases, the FS distance is far from its limit $\pi / 2$ value at every time. In the last case $(m=5)$, this distance even tends to very small values when the laser is turned off. These small FS distances illustrate the quantum compatibility of the successive active spaces at any time and induce a fast convergence of the iterative algorithm.

The failure in the case $m=2$ can be understood by comparing figures 3 and 4 Because all the evolutions starting from the $S_{o}$ subspace are calculated as a whole, we shall also look at the one issuing from the target state (cf. figure 4). The comparison with figure 3 reveals that the eight discrete time values for which the FS distance is close to $\pi / 2$ (figure 3) are identical to the time values for which the occupations of the states $(v=0, S=1)$ and $(v=5, S=1)$ decrease to zero simultaneously (figure (4). This situation is produced by the Rabi oscillations which affect the initial state 


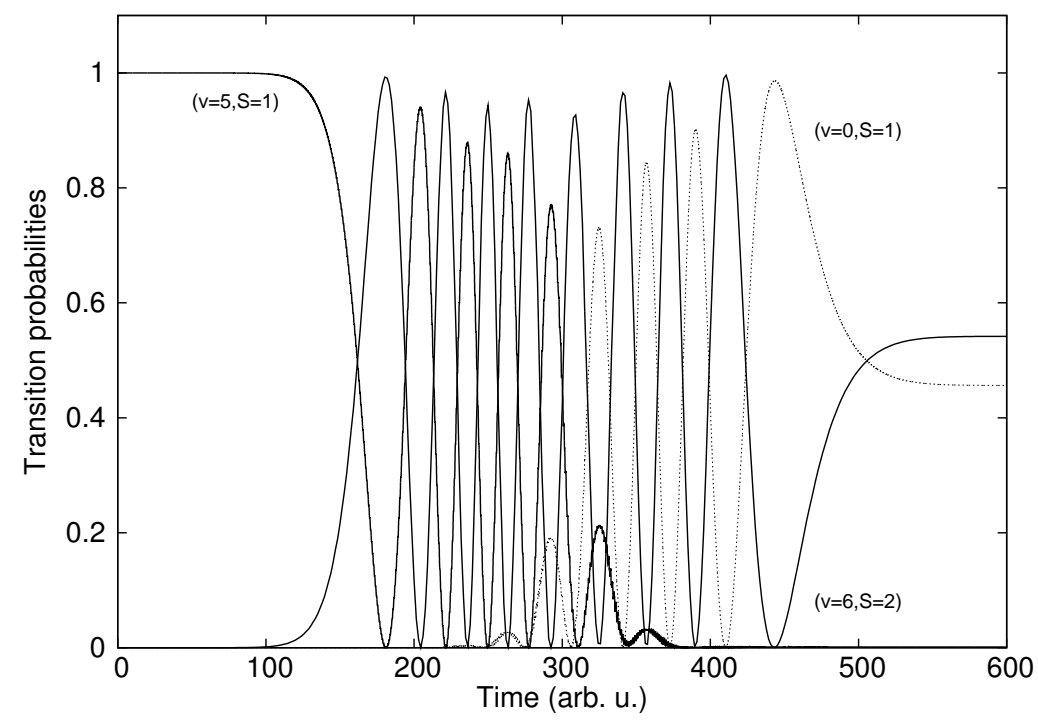

Figure 4. Populations of the three states $(v=0, S=1),(v=5, S=1)$ and $(v=6, S=2)$ when the laser field used in the STIRAP experiment (figure 2) drives a dynamics starting from the target state $(v=5, S=1)$ (full line). The evolutions of states $(v=0, S=1)$ and $(v=6, S=2)$ are represented by a dashed line and a broad full line, respectively.

$(v=5, S=1)$ for $t<300$ and the state $(v=0, S=1)$ for $t>300$ in this case. As a consequence, the $(2 \times 2)$ matrix $P_{o}^{\dagger}[U(t, 0 ; H)] P_{o}$ which leads to the FS distance (equation 24), exhibits, at these eight discrete time values, a column corresponding to the initial state $(v=5, S=1)$ equal to zero and consequently a FS distance close to $\pi / 2$. Figure 4 shows that other Rabi oscillations affect the intermediate state $(v=6, S=2)$ but the zero values of these oscillations correspond to maximum values of the oscillations affecting the initial state $(v=5, S=1)$ and the state $(v=0, S=1)$. This explains why correct results with small FS distances are obtained by using an active space of dimension $m=3$.

The best choice for the active space is the one with dimension $m=5$. This choice induces FS distances wich remain very small at all times. Moreover these distances converge to very small values when the laser field is turned off (figure 3), indicating that the wave function is, at the end, projected onto the initial $S_{o}$ subspace. In other words the dynamics is approximately cyclic within this 5 -dimensional subspace. The selection rules used to build the subspace are simple. The subspace should include the 
initial and the target state, namely $(v=0, S=1)$ and $(v=5, S=1)$ in the STIRAP experiment. The subspace should also include states which are strongly coupled to these first two ones by near resonant effects, $E(v=6, S=2)-E(v=0, S=1) \simeq \hbar \omega_{2}$ and $E(v=16, S=2)-E(v=5, S=1) \simeq \hbar \omega_{2}$. Finally the state $(v=6, S=1)$, which is not in exact resonance, has been added to these first four states. It is weakly populated during the interaction.

In the one-dimensional subspace case, the divergence of the wave operator is related to the complete population transfer between $(v=0, S=1)$ and $(v=5, S=1)$. This also produces a divergence in the effective Hamiltonian $H_{\text {eff }}(t)=P_{o} H(t) \Omega(t)$ which drives the dynamics within the active subspace. On the contrary, the use of an active subspace of dimension $m=5$, induces a $(5 \times 5) H_{\text {eff }}$ matrix whose components are always finite. The diagonal element showing the largest shift is
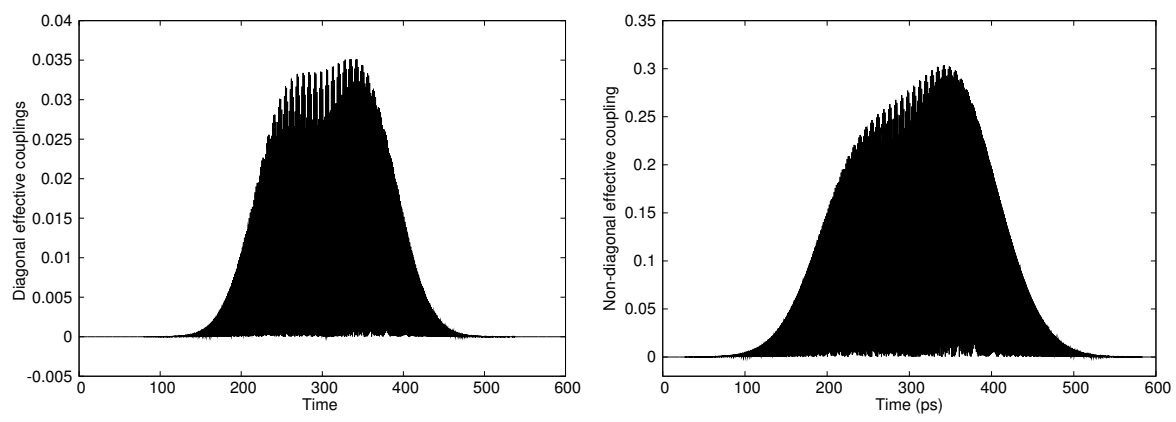

Figure 5. Shift from the non-perturbed values of the diagonal matrix elements (modulus) of $H_{\text {eff }}$, for the initial state $(v=0, S=1)$ (left frame). The right frame represents the coupling amplitude between states $(v=5, S=1)$ and $(v=6, S=2)$

the one which corresponds to the initial state $(v=0, S=1)$ and it is presented in figure (5). It exhibits relatively small modulations compared with the spacing between the non-perturbed eigenvalues, $E(v=5, S=1)-E(v=0, S=1) \simeq 5.207$ arb.u. and $E(v=6, S=2)-E(v=0, S=2) \simeq 9.984$ arb.u. Nevertheless correct solutions are obtained if, and only if these modulations are taken into account during the calculation. The non-diagonal couplings are much larger, especially the direct couplings $(v=0, S=1) \leftrightarrow(v=6, S=2)$ and $(v=6, S=2) \leftrightarrow(v=5, S=1)$ (see figure (5) ). The effective Hamiltonian $H^{\text {eff }}$ also possesses a small direct coupling between the initial $(v=0, S=1)$ and the final state $(v=5, S=1)$ (not shown). 

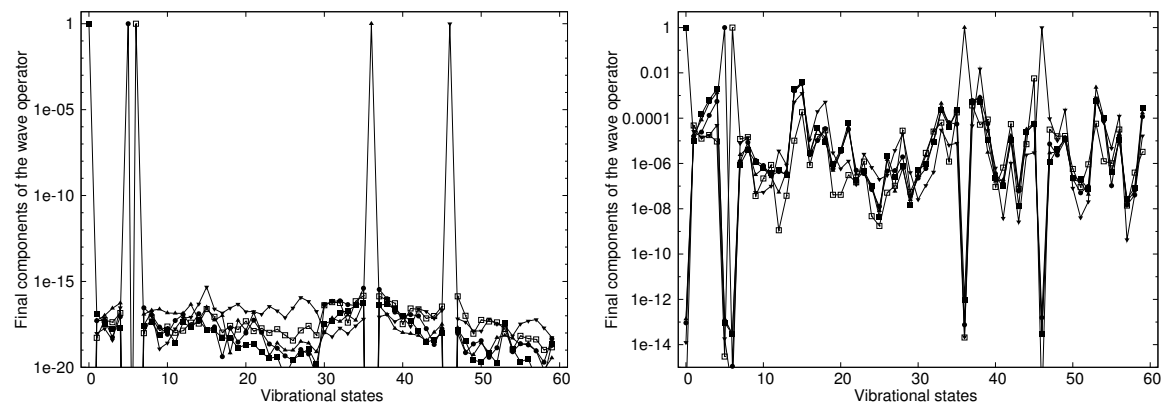

Figure 6. Component amplitudes of the five columns which constitute the wave operator at the final time $(t=T)$ when equation (3) is integrated with timedependent absorbing potential (left frame) and without time-dependent absorbing potential (right frame). Five different symbols are associated to the five initial states which compose the active space $S_{o}, \mathbf{\square} \leftrightarrow(v=0, S=1), \bullet \leftrightarrow(v=5, S=1)$, $\Delta \leftrightarrow(v=6, S=2) \boldsymbol{\nabla} \leftrightarrow(v=16, S=2), \square \leftrightarrow(v=6, S=1)$

We can analyse the cyclic character of the dynamics with respect to the selected active space with $m=5$. For doing this analysis we need to remove the artificial influence of the time-dependent absorbing potential defined in equation 4 , For a purely cyclic dynamics the solutions of eq. (3) obtained with and without time-dependent absorbing potentials would be strictly equal. The wave operator components (obtained with and without artificial absorbing potential) are shown in figure 6. Vibrational states are numbered from $v=0$ to $v=29$ for the first surface and from $v=30$ to $v=59$ for the second surface. In both frames of figure 6 , the five unit peaks correspond to the return of the wave operator to the initial active space at the end of the dynamics. The projection of $\Omega$ into the active space at $t=T$ is effectively identical to $P_{o}$ (the zero terms being approximated by small values of about $10^{-13}$ ). However in the right frame the $(5 \times 55)$ non-diagonal components coupling the active space to the complementary space take non-vanishing small values between $10^{-2}$ and $10^{-6}$, which corresponds to transition probabilities smaller than $10^{-4}$. This figure proves that the dynamics is largely (but not perfectly) cyclic within the selected 5-dimensional active space. In such a case the solution can be expanded on a basis set made from only five periodic Floquet eigenstates associated with the active space. The five eigenvalues and the initial components of the corresponding eigenvectors have been calculated using the method explained in Appendix A and are given in table 2 , These Floquet eigenstates have non-vanishing components at the two time-boundaries in the active 


\begin{tabular}{|c|c|c|c|c|c|}
\hline & \multicolumn{5}{|c|}{ Floquet eigenvalues $E_{\lambda_{j}}$} \\
\cline { 2 - 6 } & 5.6692 E-04 & -2.8759 E-03 & -2.7546 E-03 & -1.42049 E-03 & -1.6374 E-03 \\
\hline$\langle v, S|$ & \multicolumn{5}{|c|}{ Eigenvector components $\left|\left\langle v, S \mid \lambda_{j}(t=0)\right\rangle\right|$} \\
\hline$(\mathrm{v}=0, \mathrm{~S}=1)$ & 0.6531 & 0.6129 & 0.2282 & 0.3110 & 20376 E-02 \\
$(\mathrm{v}=5, \mathrm{~S}=1)$ & 0.6616 & 0.6106 & 0.1970 & 0.3875 & $2.0543 \mathrm{E}-02$ \\
$(\mathrm{v}=6, \mathrm{~S}=2)$ & 0.3674 & 0.3765 & 0.1445 & 0.8363 & $5.9142 \mathrm{E}-02$ \\
$(\mathrm{v}=16, \mathrm{~S}=2)$ & $2.4062 \mathrm{E}-02$ & 0.3321 & 0.9424 & $2.9068 \mathrm{E}-02$ & $1.9696 \mathrm{E}-03$ \\
$(\mathrm{v}=6, \mathrm{~S}=1)$ & $7.2863 \mathrm{E}-03$ & $4.0218 \mathrm{E}-03$ & $1.3086 \mathrm{E}-03$ & $6.5247 \mathrm{E}-02$ & 0.9978 \\
\hline
\end{tabular}

Table 2. The five Floquet eigenstates $\left|\lambda_{j}\right\rangle$ over which the cyclic wavefunctions can be expanded and the corresponding eigenvalues.

space exclusively. Note that these vectors and the corresponding eigenvalues depend on the Floquet Hamiltonian but also on the duration $T$ of the selected time interval.

The small defect with respect to a perfect cyclicity obtained in the waveoperator components is consistent with the results for the FS distance previously shown in figure 3 for an active space of dimension $m=5$. At time $t=600$, the laser is turned out and the FS distance is about $10^{-2}$ and not strictly equal to zero, indicating that a small part of the population is present in the complementary space.

\subsection{Dissociative dynamics of $\mathrm{H}_{2}^{+}$}

The second illustrative example is that of the $\mathrm{H}_{2}^{+}$molecule submitted to an intense laser pulse. The principal aim of this example is to test the ability of the global algorithm to describe non-adiabatic dynamics driven by a non-hermitian Hamiltonian. We only take into account the two first effective potentials [23] corresponding to the two lowest electronic states ${ }^{2} \Sigma_{g}^{+}$and ${ }^{2} \Sigma_{u}^{+}$. We make the assumption that the rotational dynamics is frozen. This is a sensible assumption because we consider only very short laser pulses. Before calculating the dynamics, the field-free Hamiltonian of $\mathrm{H}_{2}^{+}$has been pre-diagonalized on a radial grid basis using a grid method with a radial complex absorbing potential 24, 25]. A non-perturbed vibrational eigenbasis made of $2 \times 200$ eigenvalues $\varepsilon_{j}$ and bi-orthogonal eigenstates $\left\{|j\rangle,\left|j^{*}\right\rangle\right\}$ is then used (see Appendix A.4 to express the electric dipole moment operator and the corresponding matrix $\mu_{i j}$ which couples the two surfaces. Within this simple Born-Oppenheimer model, the lower 
surface supports $N_{B S}=19$ bound vibrational states. The electric field is a sum of two slightly detuned simultaneous pulses (the detuning has been adjusted to correspond to the spacing between the first two vibrational states, $\left.\omega_{1}-\omega_{2} \simeq E(v=1)-E(v=0)\right)$. We use again the expression (33) with the following parameters, given in atomic units:

$$
\left\{\begin{array}{l}
E_{1}=E_{2}=0.03 \text { a.u., } \omega_{1}=0.35 \text { a.u., } \omega_{2}=0.3398 \text { a.u. } \\
T_{1}=T_{2}=250 \text { a.u., } \tau_{1}=\tau_{2}=100 \text { a.u. }
\end{array}\right.
$$

This laser pulse is represented in figure (7). The peak value equal to $E=0.06$ a.u. corresponds to an intensity of $I=1.263 \times 10^{14} \mathrm{~W} / \mathrm{cm}^{2}$. An ample Fourier basis set of $N_{t}=2048$ functions is used to represent the interaction throughout the time interval $[0,640 \mathrm{au}]$, which is widely sufficient to include all the possible multiphoton processes expected with fields exceeding $10^{14} \mathrm{~W} / \mathrm{cm}^{2}$.

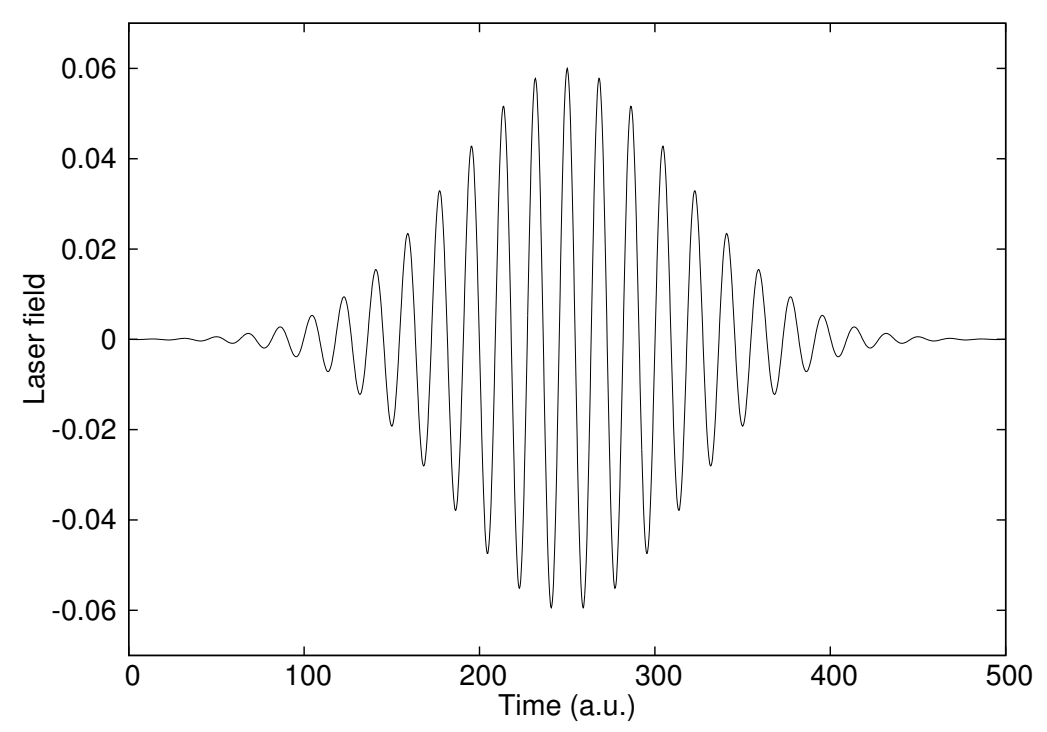

Figure 7. Laser field amplitude as a function of time

The dynamics is integrated by using an initial active space of dimension $m=41$ which includes the $N_{B S}=19$ bound states of the first surface ${ }^{2} \Sigma_{g}^{+}$, the 11 first pseudo-diffusion states which discretize the continum of this surface and the 11 last pseudo-diffusion states which discretize the continuum of the second surface ${ }^{2} \Sigma_{u}^{+}$. The dimension of this subspace is important but still small compared with the dimension of the molecular basis (here $N_{m}=400$ ). Why do we precisely choose this active subspace ? Including all the bound states in the active space is certainly a good choice. The initial wavepacket will be in general chosen as a bound wavepacket and 
after the pulse is turned off, the remaining bound wavepacket returns to this subspace. The active subspace can also be completed by including some of the discretized continuum states. The selection has been done by looking at the relative lifetimes of the different pseudo-diffusion states. States with long lifetimes may contribute to the final wavepacket and have been included in the active subspace. There are diffusion states with long lifetimes close to the dissociation limit of the first potential curve ${ }^{2} \Sigma_{g}^{+}$and other long lifetime states close to the energy trucation of the second curve ${ }^{2} \Sigma_{u}^{+}$. The complementary space is thus made of all the other pseudo-diffusion states with shorter lifetime, leading to molecular photodissociation.

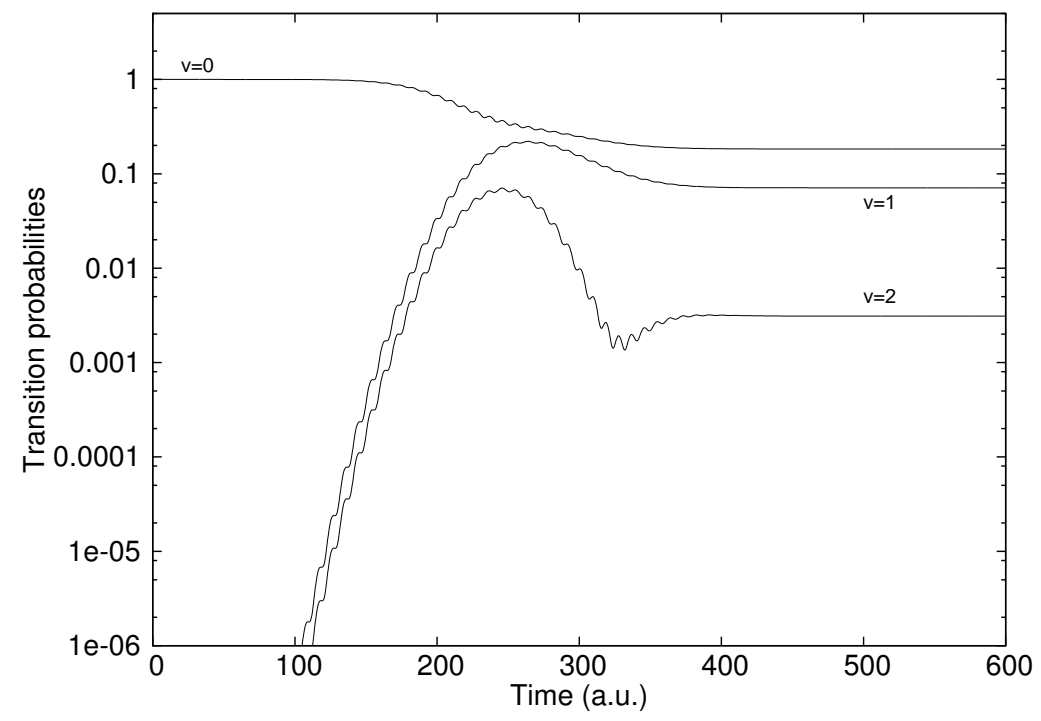

Figure 8. Transition probabilities from the initial state $(v=0)$ as a function of time, $P(v=0 \rightarrow v=0), P(v=0 \rightarrow v=1), P(v=0 \rightarrow v=2)$.

By including all the bound states in $S_{o}$, we integrate globally the transition dynamics between these bound states so that the wave operator obtained by solving eq. (3) gives us all the transition probabilities: $P_{i \rightarrow j}(t) ; i=0 \ldots 18, j=0 \ldots 18$. Two partial results are given in figures (8) and (9) for dynamics issuing from states $(v=0)$ and $(v=3)$, respectively. Moreover the various dissociation probabilities $P_{d i s s}(i)$, for an evolution issuing from the initial state $v=i$ can be obtained as

$$
P_{\text {diss }}(i)=1-\sum_{j=0}^{18} P_{i \rightarrow j}(t=T)
$$

The iterative procedure (eqs 9-201) converges after only $n=14$ iterations to $\epsilon=3.10^{-8}$, 


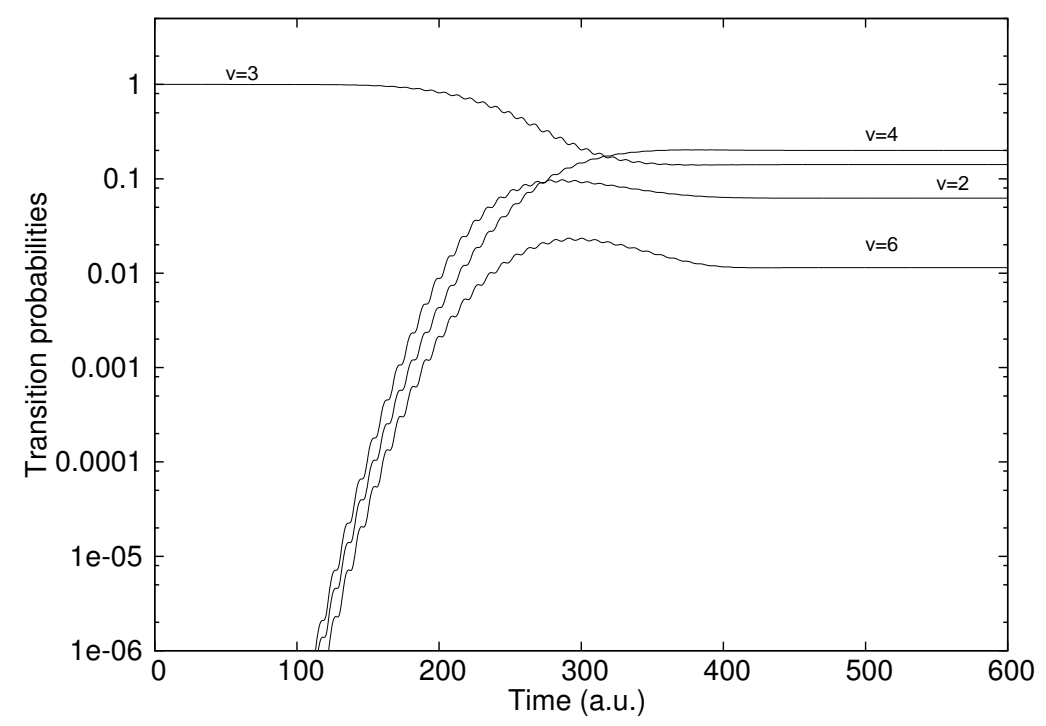

Figure 9. Transition probabilities from the initial state $(v=3)$ as a function of time, $P(v=3 \rightarrow v=2), P(v=3 \rightarrow v=3), P(v=3 \rightarrow v=4)$, $P(v=3 \rightarrow v=6)$.

\begin{tabular}{|c|c|c|c|c|c|c|c|}
\hline & \multicolumn{7}{|c|}{ Convergence factor } \\
\hline Iteration number & $\mathrm{n}=1$ & $\mathrm{n}=2$ & $\mathrm{n}=3$ & $\mathrm{n}=4$ & $\mathrm{n}=5$ & $\mathrm{n}=6$ & $\mathrm{n}=7$ \\
\hline$F_{n}^{(m=41)}$ & $5.08 \mathrm{E}-03$ & $6.01 \mathrm{E}-04$ & $1.02 \mathrm{E}-03$ & $6.81 \mathrm{E}-04$ & $2.47 \mathrm{E}-04$ & $2.75 \mathrm{E}-04$ & $1.27 \mathrm{E}-04$ \\
\hline Iteration number & $\mathrm{n}=8$ & $\mathrm{n}=9$ & $\mathrm{n}=10$ & $\mathrm{n}=11$ & $\mathrm{n}=12$ & $\mathrm{n}=13$ & $\mathrm{n}=14$ \\
\hline$F_{n}^{(m=41)}$ & $5.43 \mathrm{E}-05$ & $3.87 \mathrm{E}-05$ & $1.32 \mathrm{E}-05$ & $3.49 \mathrm{E}-06$ & $7.32 \mathrm{E}-07$ & $1.54 \mathrm{E}-07$ & $3.03 \mathrm{E}-08$ \\
\hline
\end{tabular}

Table 3. The convergence factor $F_{m}^{(n)}=\left\|\delta X^{(n)}\right\|^{2} /\left\|X^{(n)}\right\|^{2}$ (eq. 222) with respect to the iteration number $n$ for the active subspace of dimension $m=41$.

giving all the $\left(N_{B S} \times N_{B S}\right)$ transition probabilities with an accuracy of four stable digits.

The table (3) shows the convergence factor (cf eq. (22)). This good result is understandable since the active space includes all the bound states which mainly participate in the dynamics. It is true that a large part of the wave packet is projected into the two continua. But most of the pseudo-diffusion states which span these two continua have small lifetimes and their populations rapidly decrease to zero. Some long-lived diffusion states are present and can disturb the cyclic character of the dynamics but this defect is suppressed in the present treatment by including these 
states in our active subspace. As expected the populations of the long-lived diffusion states included in the active subspace states do not converge to zero at $t=T$. These results are confirmed by testing the convergence versus the composition of the active space. Including all the bound states is essential in this rather non-adiabatic example. This is the safest way to ensure an easy convergence for any dynamics issuing from bound states (for example, selecting a too small active space of dimension $m=5$ made of the first five bound states $v=0$ to $v=4$ is not sufficient and makes the iterative procedure diverge). In table 3 which corresponds to $m=41$, the convergence factor, equal to $5.08 \times 10^{-3}$ for $n=1$, converges to $3.03 \times 10^{-8}$ after $n=14$ iterations. By reducing the active space to the $m=19$ bound states, the convergence factor converges from $2.84 \times 10^{-3}$ for $n=1$ to $3.33 \times 10^{-7}$ for $n=14$. This worse result is due to non-negligible populations of some long-lived diffusion states which subsist at the end of the interaction and are worth being included in the active subspace. Increasing $n$ from 41 to 51 by adding more diffusion states does not significantly affect the results of table 3

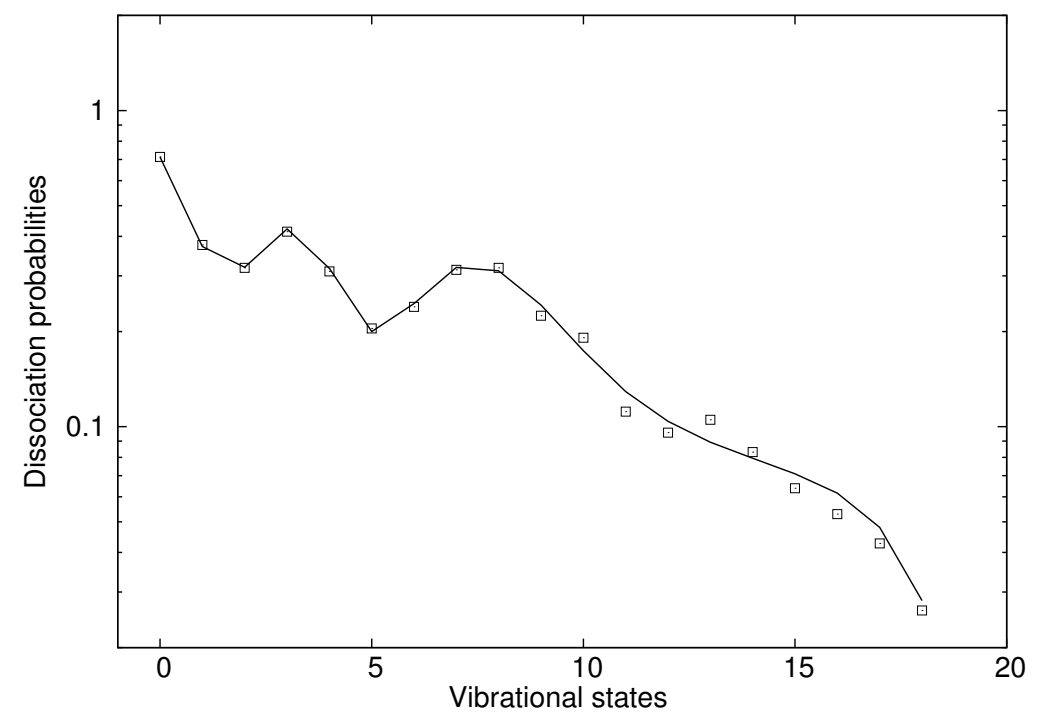

Figure 10. Dissociation probabilities associated to the initial states $(v=$ $0, \ldots v=18$ ), obtained by integrating the equation (3) in the presence of a timedependent absorbing potential (continuous line), and without absorbing potential $(\square)$.

Figure (10) displays the dissociation probabilities expressed in eq. (37). The 
nearly equal results obtained with and without absorbing potentials confirm that the dynamics is approximately cyclic with respect to the selected active subspace. This character is also confirmed by analysing the figure (11) which represents the amplitudes of the components of $X=Q_{o} \Omega$ at the final time $(t=T)$. All these components are smaller than $10^{-1}$, most of them being between $10^{-2}$ and $10^{-6}$.

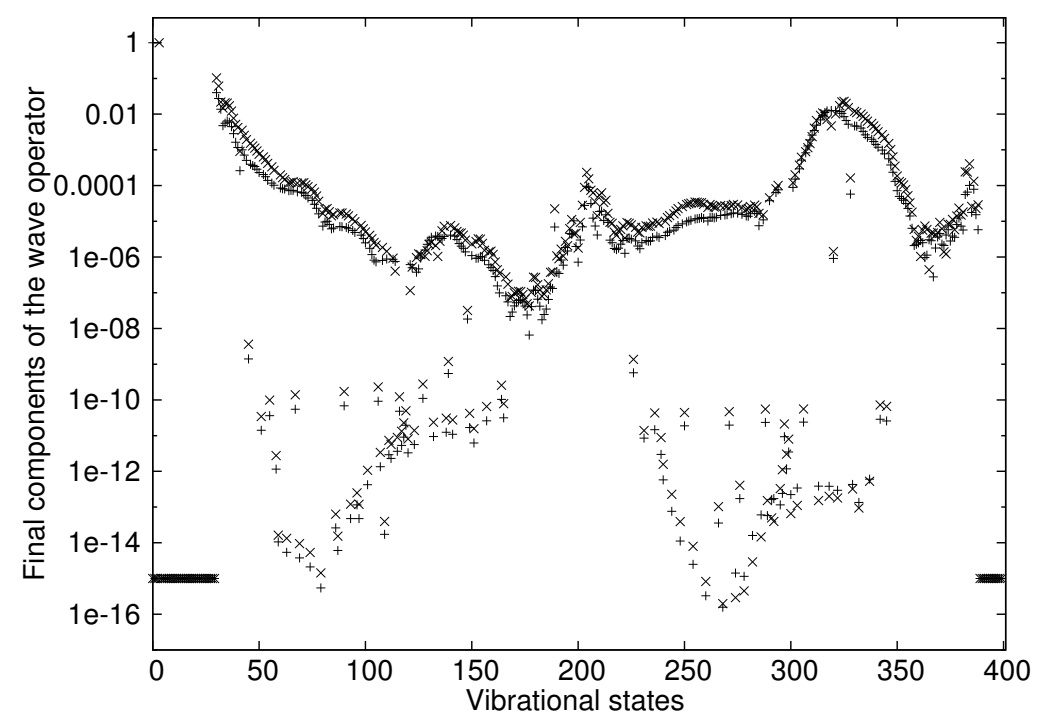

Figure 11. Wave operator component amplitudes, for the columns corresponding to the initial states $v=0(+)$ and $v=3(\times)$ at the final time $(t=T)$ when the equation (3) is solved without absorbing potential. The laser field is built on the interval $[0,500]$ a.u. and the final time is $T=640$. a.u.

\section{Conclusion}

The two illustrative examples presented in section 3 leads to the following conclusions. The multidimensional version of the global integrator significantly improves the performances of the previous one-dimensional integrator of ref. [14]. If the active space is correctly chosen, the divergences appearing in the one-dimensional case disappear and correct solutions of the Schrödinger equation with initial conditions corresponding to each one of the unperturbed molecular states which compose the initial active space are obtained, by solving only once the equation (3). In these ideal situations the convergence is fast and accurate solutions are obtained after only a few iterations.

The present theory treats both the periodic and the quasi periodic perturbations 
arising in the interactions between matter and c.w. laser fields or pulsed laser fields. It is applicable to the non-Hermitian Hamiltonians generated by using analytical continuations of the resolvent in the complex plane when $L^{2}$ representations of the continua are used. Moreover the global character of the integration algorithm makes possible the rapid repetition of perturbative calculations when some of the physical parameters (intensity, wavelength) are slightly modified. This feature is well adapted to investigate control processes which call for repeated propagation attempts.

The most delicate point is the selection of a good initial active space. Several concepts which are closely related (the cyclic character of the dynamics, the FubiniStudy distance, the time-dependent absorbing potential) participate in the selection of such a good active space, which should be as small as possible while giving a rapid convergence. In practice, looking at the coupling strength between states, including resonant and near-resonant states and long-lived continuum states, is a good guide in selecting the active space. The quality of the active space (size and choice of the relevant states) has direct consequences on the numerical convergence and can also be appreciated by monitoring a posteriori the Fubini-Study quantum distance between the initial, fixed subspace and the dynamical subspace. For example the iterative treatment diverges if this FS distance tends to $\pi / 2$ at a given arbitrary instant between $t=0$ and $t=T$.

The use of Floquet theory necessitates that the dynamics is cyclic. We have shown that (see Appendix A) in such a case (i.e. if the wave function included in the active space $S_{o}$ of dimension $m$ at $t=0$ returns to this subspace at the final time $T$ ), the wave function can be rigorously expanded on a very small Floquet eigenbasis set of dimension $m$. This is an important result which will be exploited in later work. Unfortunately, a spontaneous cyclic character is never rigorously observed. An artificial cyclic dynamics can be obtained by using a generalization of the constrained adiabatic trajectory method, i.e. by introducing an asymptotic timedependent absorbing potential (eq. (4) in order to impose the condition $X(t=T)=0$. The absorbing potential imposes the periodicity and suppresses the problems near the boundary $t=T$ (except in the case of a complete inversion with a one-dimensional active space). However it does not suppress the divergences appearing when the FS distance tends to $\pi / 2$ at intermediate instants. The best scheme for the selection of the active space is to work first without an absorbing potential by using simple selection 
criteria and then to add the absorbing potential at a second stage to suppress the small inconsistencies due to the non-perfect cyclicity. The selection criteria should take into account the distribution on the energy scale of the eigenstates dressed by the laser field.

The second illustrative example shows that our algorithm works well for dissipative systems when grouping together all the coupled bound states within the active space. In the $\mathrm{H}_{2}^{+}$case the global integrator dresses all the $N_{B S}=19$ bound states with the continua and transforms them into $N_{B S}$ resonance states which mainly participate in the dissociative dynamics.

\section{Acknowledgments}

Simulations have been executed on computers of the Utinam Institute of the Université de Franche-Comté, supported by the Région de Franche-Comté and Institut des Sciences de l'Univers (INSU).

\section{Appendix A. Cyclic evolution and Floquet basis set}

In this appendix we prove that imposing the cyclicity of the wavefunction with respect to a fixed $m$-dimensional subspace $S_{o}$ implies that only $m$ Floquet eigenvectors participate in its development (30), those vectors having non-zero components only in $S_{o}$ at $t=0$ (hence at $\left.t=T\right)$.

Appendix A.1. Cyclic wavefunction

Let $S_{o}$ be a subspace of dimension $m$ of the Hilbert space with projector

$$
P_{o}=\sum_{j=1}^{m}|j\rangle\langle j|
$$

and let $H(t)$ be the time-dependent hermitian Hamiltonian which drives the dynamics of the wave function, starting from the $S_{o}$ space, over the time interval $[0, T]$. If the evolution of the wavefunction is cyclic with respect to $S_{o}$ and if $\{|i\rangle\} i=1, \ldots m$ is a complete basis of this subspace then:

$$
\forall i \leq m \quad\left|\Psi_{i}(0)\right\rangle=|i\rangle \Rightarrow\left|\Psi_{i}(T)\right\rangle=\sum_{j=1}^{m} \Phi_{j i}|j\rangle
$$


where $\Phi$ is a unitary matrix of dimension $m$ (the basis set can be composed of the eigenvectors of the molecular Hamiltonian, $\left.H_{o}|i\rangle=e_{i}|i\rangle\right)$. If $U$ is the matrix which diagonalize the unitary matrix $\Phi$, namely

$$
\tilde{\Phi}=U \Phi U^{-1}, \text { with } \tilde{\Phi}_{j j}=e^{i \phi_{j}}
$$

with $\phi_{j}$ real, then by introducing the new basis set $|\tilde{k}\rangle=\sum_{j=0}^{m} \bar{U}_{k j}|j\rangle$ (the bar denoting the complex conjugate), one obtains

$$
\left|\Psi_{i}(T)\right\rangle=\sum_{k=1}^{m} e^{i \phi_{k}} U_{k i}|\tilde{k}\rangle
$$

\section{Appendix A.2. Expansion on the Floquet basis set}

The total interval $T$ is seen as a period for periodic Floquet eigenvectors. We assume that the Floquet spectrum is non degenerate. Using eqs.(30) and (A.4), one can write

$$
\left|\Psi_{i}(T)\right\rangle=\sum_{j} \sum_{k=1}^{m} e^{-\frac{i}{\hbar} E_{j, 0} T}\left|\lambda_{j, 0}(0)\right\rangle\left\langle\lambda_{j, 0}(0) \mid \tilde{k}\right\rangle U_{k i}
$$

Using eq. (A.4) and projecting on $\langle\tilde{k}|$ gives

$$
\left\langle\tilde{k} \mid \Psi_{i}(T)\right\rangle=e^{i \phi_{k}} U_{k i} \quad \forall k \leq m
$$

Introducing (A.5) into (A.6) leads to

$$
\sum_{j} e^{-\frac{i}{\hbar} E_{j, 0} T-i \phi_{k}}\left\langle\tilde{k} \mid \lambda_{j, 0}(0)\right\rangle\left\langle\lambda_{j, 0}(0) \mid \tilde{i}\right\rangle=\delta_{k i}
$$

By using the following notations,

$$
\left\{\begin{array}{l}
d_{j i}=\left\langle\lambda_{j, 0}(0) \mid \tilde{i}\right\rangle \\
\phi_{j k}=-\frac{1}{\hbar} E_{j, 0} T-\phi_{k}
\end{array}\right.
$$

and using the closure relation on the $\left|\lambda_{j, 0}\right\rangle$, eq. leads in the case $i=k$ to the constraint

$$
\sum_{j}\left|d_{j i}\right|^{2}\left(1-e^{i \phi_{j i}}\right)=0
$$

In each term of this sum $\left|d_{i j}\right|^{2}$ is a positive real number and $1-e^{i \phi_{j i}}$ is a complex number localized in the half plane $x>0$, on a circle tangent to the vertical axis and passing through zero only if $\phi_{i j}=0$. Consequently eq.(A.9) can be satisfied only if there exist some $j=j_{i}$ such that

$$
\left\{\begin{array}{l}
d_{j_{i} i} \neq 0 \text { and } \phi_{j_{i} i}=0 \Rightarrow \phi_{i}=\frac{1}{\hbar} E_{j, 0} T \\
d_{j i}=0, \quad \forall j \neq j_{i} .
\end{array}\right.
$$


If two or more terms among the $d_{j i}$ were different from zero, this would imply that the corresponding phases $\phi_{j i}$ are simultaneously zero, which is impossible since we have assumed a non-degenerate Floquet spectrum.

In the case $i \neq k$, eq. A.7 reads

$$
\sum_{j} e^{-i \phi_{j k}} \bar{d}_{j k} d_{j i}=0
$$

Considering eq.(A.10), we see that $d_{j k}=0$ except when $j=j_{k}$, and the same is true for $d_{j i}=0$ except when $j=j_{i}$. Then eq. (A.11) implies that

$$
i \neq k \Rightarrow j_{i} \neq j_{k} \text {. }
$$

Finally the results (A.10) and (A.12) prove that only $m$ periodic Floquet eigenvectors participate in the wave function expansion with the conditions $P_{o}\left|\lambda_{j_{i}, 0}(0)\right\rangle=\left|\lambda_{j_{i}, 0}(0)\right\rangle$. By introducing a new numbering of the basis set such that $j_{i} \rightarrow i$ we obtain

$$
\left\{\begin{array}{l}
\left|\Psi_{i}(t)\right\rangle=\sum_{j=1}^{m} e^{-\frac{i}{\hbar} E_{j, 0} t}\left|\lambda_{j, 0}(t)\right\rangle U_{j i} \\
U_{j i}=\langle\tilde{j} \mid i\rangle=\left\langle\lambda_{j, 0}(0) \mid i\right\rangle
\end{array}\right.
$$

Appendix A.3. Generalized Floquet state components from the wavefunction

Once the evolution operator is obtained, it is possible to deduce the initial and final components of the $m$ generalized Floquet vectors which participates in the $\Psi_{i}$ expansion. By using the T-periodicity of Floquet eigenvectors, A.13) at initial and final time $T$ gives

$$
\begin{aligned}
& P_{o}\left|\Psi_{i}(0)\right\rangle=|i\rangle=\sum_{j=1}^{m} P_{o}\left|\lambda_{j, 0}(0)\right\rangle U_{j i} \\
& P_{o}\left|\Psi_{i}(T)\right\rangle=\sum_{j=1}^{m} e^{-\frac{i}{\hbar} E_{j, 0} T} P_{o}\left|\lambda_{j, 0}(0)\right\rangle U_{j i} .
\end{aligned}
$$

Using $(m \times m)$ matrices with $\boldsymbol{\Psi}_{k i}=\left\langle k \mid \Psi_{i}(T)\right\rangle, \boldsymbol{\Lambda}_{k i}=\left\langle k \mid \lambda_{i, 0}(0)\right\rangle, \mathbf{E}_{k i}=e^{-\frac{i}{\hbar} E_{k, 0} T} \delta_{k i}$, $U$ defined in (A.3) and the identity matrix $I$, we obtain

$$
\left\{\begin{array}{l}
\Psi=\Lambda \mathbf{E} U \\
I=\Lambda U
\end{array}\right.
$$

which leads to the following result:

$$
\mathbf{\Psi}=\mathbf{\Lambda} \mathbf{E} \boldsymbol{\Lambda}^{-1} \text {. }
$$

Initial (and final) components of the $m$ generalized Floquet states of interest within the subspace $S_{o}$ and the associated Floquet eigenvalues can then be calculated by diagonalizing the small matrix $\boldsymbol{\Psi}$. 


\section{Appendix A.4. Non-hermitian case}

The above reasoning can be followed also in the case of a non-hermitian Hamiltonian accounting for dissipative system such as photodissociation problems. Biorthonormal basis sets with left and right eigenvectors must be introduced for both the molecular Hilbert space,

$$
\begin{aligned}
& H_{0}|j\rangle=\varepsilon_{j}|j\rangle, \\
& H_{0}^{\dagger}\left|j^{*}\right\rangle=\bar{\varepsilon}_{j}\left|j^{*}\right\rangle,
\end{aligned}
$$

and for the Floquet Hamiltonian,

$$
\begin{aligned}
& H_{F}\left|\lambda_{j, 0}\right\rangle=E_{j, 0}\left|\lambda_{j, 0}\right\rangle, \\
& H_{F}^{\dagger}\left|\lambda_{j, 0}^{*}\right\rangle=\bar{E}_{j, 0}\left|\lambda_{j, 0}^{*}\right\rangle .
\end{aligned}
$$

In this case the wavefunction expansion on the generalized Floquet eigenvectors is

$$
\left|\Psi_{i}(t)\right\rangle=\sum_{j} e^{-i E_{j, 0} t / \hbar}\left|\lambda_{j, 0}(t)\right\rangle\left\langle\lambda_{j, 0}^{*}(0) \mid i\right\rangle
$$

Since the Floquet Hamiltonian is non-hermitian but still symmetric (as is the case in the second application concerning the $\mathrm{H}_{2}^{+}$molecule), the normalization of the left eigenvectors can be chosen as

$$
\left|\lambda_{j, 0}^{*}\right\rangle=\overline{\left|\lambda_{j, 0}\right\rangle}
$$

The wavefunction is supposed to be cyclic with respect to the subspace $S_{o}$ with projector $P_{o}=\sum_{j=1}^{m}|j\rangle\left\langle j^{*}\right|$. This means that the following condition is satisfied:

$$
\forall i \leq m \quad\left|\Psi_{i}(0)\right\rangle=|i\rangle \Rightarrow|\Psi(T)\rangle=\sum_{j=1}^{m} \hat{\Phi}_{j i}|j\rangle
$$

where $\hat{\Phi}$ is now a non-unitary matrix of dimension $m$. Assuming that $\hat{\Phi}$ remains diagonalizable ( $\hat{U}$ being the eigenvector matrix) and using the c-product normalization condition for the left eigenvectors [19] $\left(\left|j^{*}\right\rangle=|\bar{j}\rangle\right)$, the above reasoning leads to a different constraint for the components of the Floquet eigenvectors:

$$
\sum_{j} e^{-\frac{i}{\hbar} E_{j, 0} T-i \phi_{k}}\left\langle\tilde{k}^{*} \mid \lambda_{j, 0}(0)\right\rangle\left\langle\lambda_{j, 0}^{*}(0) \mid \tilde{i}\right\rangle=\delta_{k i},
$$

with $E_{j, 0}$ and $\phi_{k}$ complex. This equation is equivalent to

$$
\begin{aligned}
& \sum_{j} d_{j i}^{2}\left(1-e^{i \phi_{j i}}\right)=0 \quad \text { if } i=k \\
& \sum_{j} e^{i \phi_{j k}} d_{j k} d_{j i}=0 \quad \text { if } i \neq k
\end{aligned}
$$


with

$$
\left\{\begin{array}{l}
d_{j i}=\left\langle\lambda_{j, 0}^{*}(0) \mid \tilde{i}\right\rangle \in \mathbb{C} \\
\phi_{j k}=-\frac{1}{\hbar} E_{j, 0} T-\phi_{k} \in \mathbb{C},
\end{array}\right.
$$

A solution similar to the one obtained in the hermitian case (A.3) still holds, with all the $d_{i j}=0$ except for a particular value $j=j_{i}$ and finally

$$
\left\{\begin{array}{l}
\left|\Psi_{i}(t)\right\rangle=\sum_{j=1}^{m} e^{-\frac{i}{\hbar} E_{j, 0} t}\left|\lambda_{j, 0}(t)\right\rangle \hat{U}_{j i} \\
\hat{U}_{j i}=\left\langle\tilde{j}^{*} \mid i\right\rangle=\left\langle\lambda_{j, 0}^{*}(0) \mid i\right\rangle .
\end{array}\right.
$$

However eq. A.23) leaves open the possibility of accidental solutions with several non-zero components $d_{i j}$ and it is not possible to prove that the above solution is unique.

\section{References}

[1] Shapiro M, Brumer P 2003 Principles of the quantum control of molecular processes. (Wiley interscience. Hoboken, New Jersey)

[2] Werschnik J and Gross E.K.U 2007 J Phys B 40 R175-R211

[3] Shirley J H 1965 Phys. Rev. B 138, 979

[4] Sambe H 1973 Phys. Rev. A 7, 2203

[5] Bialynicki-Birula I and Bialinicka-Birula Z 1976 Phys. Rev. A14, 1101, Bialynicki-Birula and Van C L 1980 Acta Physica Polonica A57, 599

[6] Guérin S. and Jauslin H.R. 2003 Advances in Chem. Phys. 125, 147

[7] Leforestier C et al 1991 J. Comp. Phys. 94, 59

[8] Balakrishnan N, Kalyanaraman C and Sathyamurthy N 1997 Phys. Rep. 280, 79

[9] Kosloff D and Kosloff R 1983 J. Comp. Phys. 52, 35

[10] Jolicard G, Viennot D and Killingbeck JP 2004 J. Phys. Chem. A 108, 8580-8589

[11] Leclerc A, Guérin S, Jolicard G and Killingbeck JP 2011 Phys. Rev. A 83,032113

[12] Vitanov N V Halfmann T Shore B W Bergmann K 2001 Annu. Rev. Phys. Chem. 52763

[13] Jolicard G and Killingbeck J P 2003 J. Phys. A 36 R411-R473

[14] Leclerc A and Jolicard G 2015 J. Phys. A: Math. Theor. 48, 225205

[15] Viennot D 2014 J. Phys. A. 47065302

[16] Viennot D, Jolicard G. and Killingbeck J.P. 2006 J. Phys A39, 7065

[17] Aharonov Y. and Anandan J. 1987 Phys. Rev. Lett.58, 1593

[18] Chu I. 1989 Adv. Chem. Phys.73, 739

[19] Moiseyev N 2011 Non-Hermitian Quantum Mechanics (Cambridge University Press, Cambridge)

[20] Chenel A, Drive G, Meier C and Desouter-Lecomte M 2012 J. Phys. ChemA 116, 11273

[21] Viennot D. 2007 J. Math. Phys48, 052102

[22] Shore B W 2008 Acta Physica Slovaca 58, 243-486

[23] Bunkin F and Tugov I 1973Phys. Rev. A8, 601

[24] Marston C C and Balint-Kurti G G 1989 J. Chem. Phys. 913571 
[25] Poirier B and Carrington T 2003J. Chem. Phys.118 17 